\title{
BMJ Open Rationale and design of the ADAPT- TAVR trial: a randomised comparison of edoxaban and dual antiplatelet therapy for prevention of leaflet thrombosis and cerebral embolisation after transcatheter aortic valve replacement
}

To cite: Park H, Kang D-Y, Ahn J-M, et al. Rationale and design of the ADAPTTAVR trial: a randomised comparison of edoxaban and dual antiplatelet therapy for prevention of leaflet thrombosis and cerebral embolisation after transcatheter aortic valve replacement. BMJ Open 2021;11:e042587. doi:10.1136/ bmjopen-2020-042587

- Prepublication history and supplemental material for this paper is available online. To view these files, please visit the journal online (http://dx.doi. org/10.1136/bmjopen-2020042587).

HP and D-YK contributed equally.

HP and D-YK are joint first authors.

Received 12 July 2020 Revised 14 November 2020 Accepted 04 December 2020

Check for updates

(c) Author(s) (or their employer(s)) 2021. Re-use permitted under CC BY-NC. No commercial re-use. See rights and permissions. Published by BMJ.

For numbered affiliations see end of article.

Correspondence to

Dr Duk-Woo Park;

dwpark@amc.seoul.kr

Hanbit Park, ${ }^{1}$ Do-Yoon Kang, ${ }^{1}$ Jung-Min Ahn, ${ }^{1}$ Kyung Won Kim, ${ }^{2,3}$ Anthony Y T Wong, ${ }^{4}$ Simon C C Lam, ${ }^{4}$ Wei-Hsian Yin, ${ }^{5}$ Jeng Wei, ${ }^{5}$ Yung-Tsai Lee, ${ }^{6}$ Hsien-Li Kao, ${ }^{6}$ Mao-Shin Lin, ${ }^{6}$ Tsung-Yu Ko, ${ }^{7}$ Won-Jang Kim, ${ }^{8}$ Se Hun Kang, ${ }^{8}$ Euihong Ko, ${ }^{1}$ Dae-Hee Kim, ${ }^{1}$ Hyun Jung Koo, ${ }^{3}$ Dong Hyun Yang, ${ }^{3}$ Joon-Won Kang, ${ }^{3}$ Seung Chai Jung, ${ }^{3}$ Jae-Hong Lee, ${ }^{9}$ Sung-Cheol Yun, ${ }^{10}$ Seung-Jung Park, ${ }^{1}$ Duk-Woo Park (1) ${ }^{1}$

\section{ABSTRACT}

Introduction Optimal antithrombotic strategy following transcatheter aortic valve replacement (TAVR) is still unknown. We hypothesised that the direct factor Xa inhibitor edoxaban can potentially prevent subclinical leaflet thrombosis and cerebral embolisation compared with conventional dual antiplatelet therapy (DAPT) in patients undergoing TAVR.

Methods and analysis The ADAPT-TAVR trial is an international, multicentre, randomised, open-label, superiority trial comparing edoxaban-based strategy and DAPT strategy in patients without an indication for oral anticoagulation who underwent successful TAVR. A total of 220 patients are randomised (1:1 ratio), 1-7 days after successful TAVR, to receive either edoxaban $(60 \mathrm{mg}$ daily or $30 \mathrm{mg}$ daily if patients had dose-reduction criteria) or DAPT using aspirin (100 mg daily) plus clopidogrel (75 mg daily) for 6 months. The primary endpoint was an incidence of leaflet thrombosis on four-dimensional, volume-rendered cardiac CT imaging at 6 months postTAVR. The key secondary endpoints were the number of new lesions and new lesion volume on brain diffusionweighted MRI and the changes in neurological and neurocognitive function assessment between immediate post-TAVR and 6 months of study drug administration. Detailed clinical information on thromboembolic and bleeding events were also assessed.

Ethics and dissemination Ethic approval has been obtained from the Ethics Committee/Institutional Review Board of Asan Medical Center (approval number: 20171317) and this trial is also approved by National Institute of Food and Drug Safety Evaluation of Republic of Korea (approval number: 31511$)$. Results of this study will be disseminated in scientific publication in reputed journals.

\section{Strengths and limitations of this study}

- The Anticoagulation versus Dual Antiplatelet Therapy for Prevention of Leaflet Thrombosis and Cerebral Embolisation after Transcatheter Aortic Valve Replacement (ADAPT-TAVR) trial is a multinational, multicentre, prospective, randomised, open-label, superiority trial comparing efficacy and safety of edoxaban versus DAPT with aspirin plus clopidogrel in patients undergoing TAVR.

- The primary endpoint is an incidence of leaflet thrombosis on 4-D, volume-rendered cardiac CT at 6 months post-TAVR and the key secondary endpoints are cerebral embolisation (documented with brain MRI) and neurological and neurocognitive function.

- This trial is planned to complete the 3-year enrolment period for the prespecified 220 subjects from the five participating centres.

- This trial will provide important clinical insights on edoxaban-based anticoagulation strategy compared with DAPT strategy post-TAVR with respect to leaflet thrombosis and associated cerebral embolisation and neurocognitive function.

- This trial may be underpowered to detect any clinically relevant differences in clinical outcomes between two treatment strategies.

Trial registration number NCT03284827.

\section{INTRODUCTION}

Transcatheter aortic valve replacement (TAVR) has been positioned as a valuable 
treatment option for patients with symptomatic severe aortic stenosis (AS) who are at inoperable, high or intermediate risk for conventional surgical aortic valve replacement (SAVR), on the basis of clinical evidence from multiple randomised clinical trials (RCTs) ${ }^{1-7}$ Recently, TAVR has become a valid alternative to SAVR even in patients at low surgical risk. ${ }^{8}$ Despite of such proven efficacy and safety of TAVR in patients with severe AS at diverse surgical risks, thromboembolic complications (stroke, systemic embolism, valve thrombosis and venous thromboembolism) have been observed post-TAVR. In addition, observational data reported that subclinical leaflet thrombosis and reduced leaflet motion of bioprosthetic aortic valves have been documented by fourdimensional $\mathrm{CT}^{10}{ }^{10}$ and the presence of subclinical leaflet thrombosis might be associated with increased rates of stroke or transient ischaemic attacks (TIAs). ${ }^{11-13}$ Despite excellent outcomes after TAVR with newer-generation valves, prevention and optimal management of subclinical leaflet thrombosis can offer a potential opportunity for further improvement in valve haemodynamics and durability. ${ }^{14}$

In routine clinical practice, optimal post-TAVR antithrombotic therapy is still controversial and a practice variation of antithrombotic regimens is substantially high without strong evidences for their recommendations. ${ }^{15}$ Empirically, dual antiplatelet therapy (DAPT) of aspirin plus clopidogrel has been used for at least 6 months after TAVR, ${ }^{1-9}$ although such recommendation was based mainly on expert consensus. After several studies reported that valve thrombosis developed in patients receiving antiplatelet therapy alone but not in those receiving oral anticoagulation (OAC) therapy, ${ }^{10}{ }^{11}$ updated guidelines recommend that $\mathrm{OAC}$ with vitamin $\mathrm{K}$ antagonist (VKA) may be a reasonable approach for at least 3 months after TAVR in patients at low risk of bleeding (class IIb). ${ }^{16}$ However, clinical evidence to support this recommendation are still lacking (level of evidence B-NR: data were derived from one or more non-randomised trials or metaanalysis of such studies).

Edoxaban once daily is a well-tolerated inhibitor of factor Xa that has demonstrated a superior safety with non-inferior efficacy compared with VKA for prevention of stroke or systemic embolisation or recurrent symptomatic venous thromboembolism in diverse clinical settings. ${ }^{17}{ }^{18}$ We hypothesise that edoxaban, a non-VKA oral anticoagulant (NOAC), potentially reduces the risk of subclinical leaflet thrombosis and cerebral embolisation compared with conventional DAPT-based strategy in patients undergoing TAVR. The Anticoagulation versus DAPT for Prevention of Leaflet Thrombosis and Cerebral Embolisation after Transcatheter Aortic Valve Replacement (ADAPT-TAVR) trial is a multicentre, randomised, open-label, active-treatment, controlled trial to compare the efficacy of edoxaban and DAPT for prevention of leaflet thrombosis documented by high-resolution fourdimensional (4-D) cardiac CT and cerebral emboliszation documented by brain MRI and associated neurological and neurocognitive function in patients who underwent successful TAVR procedure.

\section{METHODS AND ANALYSIS}

Trial design and objectives

The ADAPT-TAVR trial is a multinational, multicentre, prospective, randomised, open-label, superiority trial that compared the efficacy of a strategy of OAC with edoxaban and DAPT with aspirin plus clopidogrel in patients without an indication for chronic anticoagulation who underwent successful TAVR for symptomatic severe AS (figure 1). The trial is being conducted in five major centres in three countries (The Republic of Korea, Hong Kong and Taiwan).

The primary objective of ADAPT-TAVR is to demonstrate the superiority of a NOAC strategy with edoxaban (experimental arm) as compared with the current standard of care DAPT (control arm) in the prevention of leaflet thrombosis (documented by 4-D cardiac CT). The main secondary objective is to compare the two antithrombotic strategies with regard to the potential risk of cerebral embolisation (documented with brain MRI) and the changes in neurological and neurocognitive function. Other objectives for clinical assessment are to investigate the time from randomisation to the first occurrence of efficacy and safety clinical outcomes including death, myocardial infarction (MI), stroke or TIAs, or bleeding events.

\section{Study population}

Patients aged $\geq 18$ years with severe symptomatic AS who underwent successful TAVR procedure (either native valve or valve in valve) with any approved/marketed device (ie, SAPIEN 3, Evolut R or Evolut PRO) were eligible for participation in the trial. A successful TAVR procedure was defined according the Valve Academic Research Consortium-2 (VARC-2) criteria as follows ${ }^{19}$ : (1) correct position of a single prosthetic heart valve into the proper anatomical location; (2) intended performance of the prosthetic heart valve with presence of all 3 of the following conditions post-TAVR (1. mean aortic valve gradient $<20 \mathrm{~mm} \mathrm{Hg}$, 2. peak transvalvular velocity $<3.0$ $\mathrm{m} / \mathrm{s}$ and 3. no moderate or severe aortic valve regurgitation) and (3) absence of periprocedural major complications (any type of stroke, life-threatening bleeding, acute coronary artery obstruction requiring intervention, major vascular complication requiring intervention, unresolved acute valve thrombosis or any requirement of a repeat procedure). The key exclusion criteria were any established indication for long-term anticoagulation (eg, concomitant atrial fibrillation) and any absolute indication for DAPT (eg, recent acute coronary syndromes or recent or concomitant percutaneous coronary intervention) at the time of screening. Detailed information on inclusion and exclusion criteria is listed in box 1 .

\section{Randomisation and treatment groups}

Eligible patients who met the study inclusion criteria and met none of the exclusion criteria are randomly (1:1 ratio) 


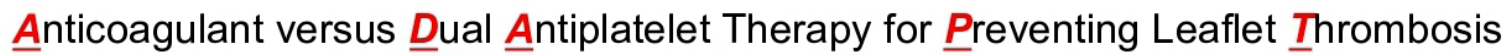
After $\underline{T}$ ranscatheter $\underline{A}$ ortic $\underline{\text { Valve }} \underline{\underline{R}}$ eplacement

\section{ADAPT-TAVR Trial}

\section{0 patients after successful TAVR procedure}

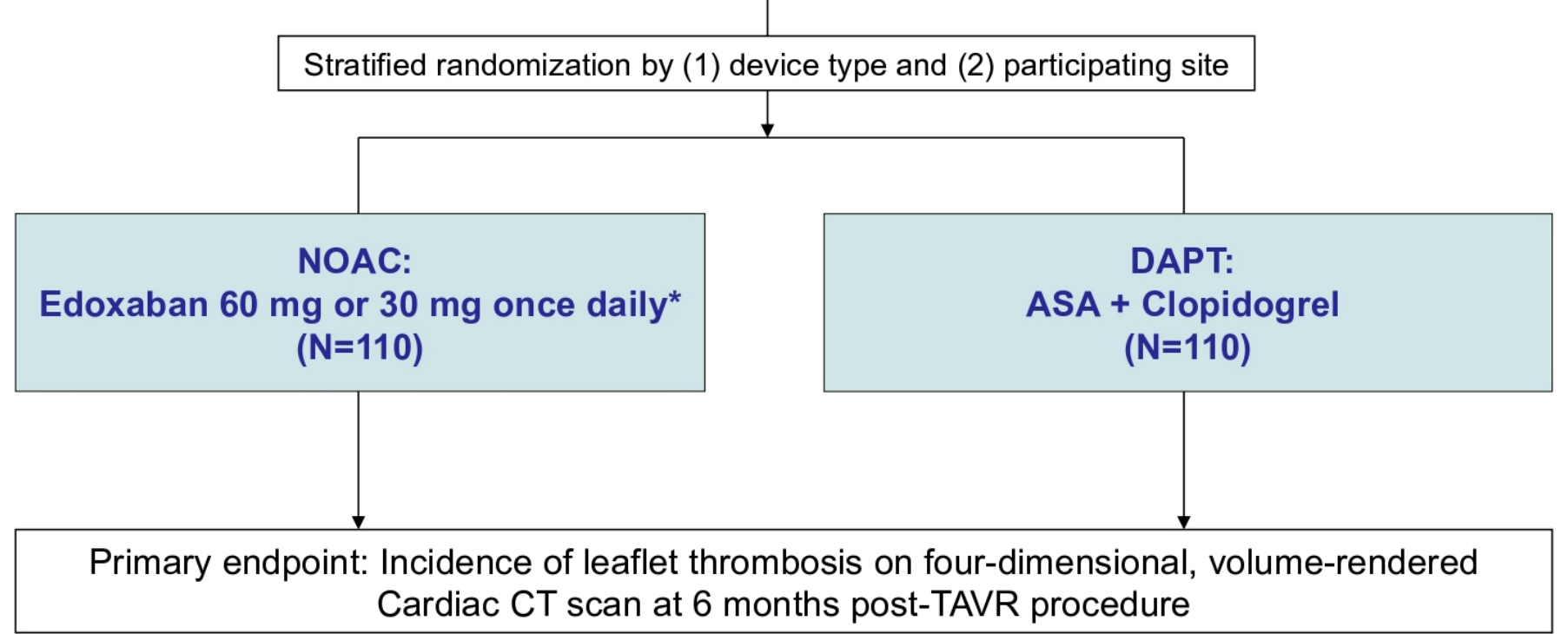

Figure 1 Study flow diagram. Successful TAVR as defined in the 'study population and methods' section. * $30 \mathrm{mg}$ once daily if moderate or severe renal impairment (creatinine clearance $15-50 \mathrm{~mL} / \mathrm{min}$ ), low body weight $\leq 60 \mathrm{~kg}$ or concomitant use of P-glycoprotein inhibitors (cyclosporin, dronedarone, erythromycin, ketoconazole). ASA, aspirin; DAPT, dual antiplatelet therapy; NOAC, non-vitamin K antagonist oral anticoagulant.

assigned to receive either (1) NOAC with edoxaban (60 $\mathrm{mg}$ once daily or $30 \mathrm{mg}$ once daily with dose-reduction criteria) or (2) DAPT with aspirin (100 mg once daily) plus clopidogrel (75 mg once daily) for 6 months after successful TAVR. Central randomisation is performed with the use of an Interactive Web Response System and stratified by type of TAVR valve (balloon-expandable or self-expandable) and participating centre with block sizes of 4 or 6 . Randomisation is performed after successful TAVR when the patient has stabilised ( 1 to 7 days after index TAVR procedure) and before hospital discharge. Duration of study drug treatment and subject follow-up will be at least 6 months.

In patients assigned to the edoxaban group (experimental arm), the investigational product is open-labelled edoxaban $60 \mathrm{mg}$ or $30 \mathrm{mg}$ tablet taken orally once daily for 6 months. Edoxaban is started at the time of randomisation and irrespective of the pre-existing antithrombotic regimen. Edoxaban $30 \mathrm{mg}$ tablet orally once daily is given for randomised patients with the following dosereduction criteria: (1) body weight $\leq 60 \mathrm{~kg}$, (2) moderate to severe renal impairment (defined as a calculated creatinine clearance (Cockroft-Gault formula) between 15 and $50 \mathrm{~mL} / \mathrm{min}$ ) or (3) concomitant P-glycoprotein inhibitors (cyclosporine, dronedarone, erythromycin or ketoconazole). Patients assigned to the DAPT group (control arm) will receive aspirin $100 \mathrm{mg}$ and clopidogrel $75 \mathrm{mg}$ once daily. Naïve patients will initially be loaded with aspirin (200 mg) and clopidogrel (300 mg) according to local practice. After 6 months of study medications in both groups, patients will continue to use lowdose aspirin $(100 \mathrm{mg})$ alone indefinitely.

In case new-onset atrial fibrillation (NOAF) occurs after randomisation, given that the potential thromboembolic risk of NOAF after TAVR is substantial, ${ }^{20}$ full OAC will be implemented with maintenance of the original treatment assignment. In the edoxaban group, the assigned treatment remains as the protocol. In the DAPT group, use of VKA or NOAC was allowed at the treating physician's discretion. Because this protocol adaptation is an integral part of the study protocol regimens, endpoints occurring under post-NOAF study treatments are retained in the primary study analysis (intention-to-treat principle).

\section{Study endpoints and follow-up}

The primary and secondary endpoints of the ADAPTTAVR trial are listed in box 2. The primary study endpoint is an incidence of leaflet thrombosis on 4-D, 


\section{Box 1 Inclusion and exclusion criteria}

\section{Inclusion criteria}

1. Patients aged $\geq 18$ with symptomatic aortic stenosis who underwent successful transcatheter aortic valve replacement (TAVR) pro-

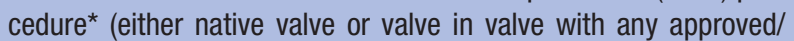
marketed device).

${ }^{*}$ A successful TAVR is defined as device success according to the Valve Academic Research Consortium-2 criteria $^{19}$ :

(1) Correct positioning of a single prosthetic heart valve into the proper anatomical location.

(2) Intended performance of the prosthetic heart valve (no prosthesis-patient mismatch and mean aortic valve gradient $<20 \mathrm{~mm}$ $\mathrm{Hg}$ or peak velocity $<3 \mathrm{~m} / \mathrm{s}$, no moderate or severe prosthetic valve regurgitation.

(3) Absence of periprocedural complications (any type of stroke, life-threatening bleeding, acute coronary artery obstruction requiring intervention, major vascular complication requiring intervention, unresolved acute valve thrombosis, or any requirement of a repeat procedure).

2. The patient or guardian agrees to the study protocol and the schedule of clinical follow-up, and provides informed, written consent, as approved by the appropriate institutional review board/ethical Committee of the respective clinical site.

\section{Exclusion criteria}

1. Any atrial fibrillation with an indication for chronic oral anticoagulation (OAC).

2. An ongoing indication for $\mathrm{OAC}$ or any other indication for continued treatment with any $\mathrm{OAC}$.

3. Any ongoing indication for dual antiplatelet therapy (recent acute coronary syndrome or percutaneous coronary intervention within 12 months).

4. Planned coronary or vascular intervention or major surgery.

5. The risk of bleeding increased due to the following reasons at the time of TAVR procedure:

- History of gastrointestinal ulcers within 1 month.

- Malignant tumortumour with high risk of bleeding.

- Brain or spinal cord injury within 1 month.

- History of intracranial or intracerebral haemorrhagehaemorrhage within 12 months.

- Oesophageal varices.

- Arteriovenous malformations.

- Vascular aneurysms.

- Spinal or intracerebral vascular abnormalities.

- Active bleeding.

- Haemoglobin level $<7.0 \%$ or platelet count $\leq 50 \times 10^{9} / \mathrm{L}$

- History of major surgery within 1 month.

6. Clinically overt stroke within the last 3 months.

7. Moderate and severe hepatic impairment, and any hepatic disease associated with coagulopathy.

8. Severe renal impairment (creatinine clearance by Cockcroft-Gault equation $<30 \mathrm{~mL} / \mathrm{min}$ per $1.73 \mathrm{~m}^{2}$ ), chronic dialysis or post-TAVR unresolved acute kidney injury.

9. Terminal illness with life expectancy $<6$ months.

10. History of hypersensitivity to edoxaban, aspirin or clopidogrel.

11. Severe hypertension.

12. Prosthetic heart valve replacement for which anticoagulant therapy is essential.

13. Moderate to severe mitral stenosis.

14. Pulmonary embolism requiring thrombolysis or pulmonary embolectomy.

Continued
Box 1 Continued

15. Active participation in another drug or device investigational study, which was not completed in the primary endpoint follow-up period.

16. Pregnancy test results are positive (all pregnant women should undergo urinary human chorionic gonadotropin testing within 7 days days prior to screening and/or randomisation) or during pregnancy or lactation.

17. Genetic problem with galactose intolerance, Lapp lactase deficiency or glucose-galactose malabsorption.

18. Current or history of aspirin-induced or nonsteroidal antiinflammatory drugs (NSAIDs)-induced asthma.

19. Haemophilia.

20. Use of methotrexate at doses of $\geq 15 \mathrm{mg}$ per week.

21. Unsuitable condition to undergo brain MRI and/or cardiac CT (eg, tremor from Parkinson's disease). This is at the discretion of the investigators.

volume-rendered cardiac CT at 6 months post-TAVR. The key secondary endpoints for assessment of cerebral embolisation, which was assessed by the number of new lesions and new lesion volume on brain MRI scans at 6 months relative to immediate post-TAVR, and the new changes of neurological and neurocognitive function assessment between post-TAVR and 6 months of study drug administration. Other secondary endpoints for assessment of ischaemic and bleeding complications includes death (all-cause, cardiovascular or non-cardiovascular), MI, stroke (disabling or non-disabling) or TIAs, or bleeding events (life-threatening or disabling, major bleeding or minor). Serial echocardiographic parameters (the mean transaortic valve pressure gradient and velocity time integral ratio) are also assessed at baseline, postprocedure, and 6-month follow-up. All clinical endpoints are adjudicated according to VARC-2 criteria $^{21}$ and the Neurologic

\section{Box 2 Primary and secondary endpoints}

\section{Primary endpoint}

Incidence of leaflet thrombosis on four-dimensional, volume-rendered cardiac CT imaging at 6 months post-transcatheter aortic valve replacement (TAVR) procedure

Secondary endpoints*

1. Number of new lesions on brain MRI scans at 6 months relative to immediate post-TAVR.

2. New lesion volume on brain MRI.

3. Neurological and neurocognitive function.

4. Echocardiographic parameters (mean transaortic valve pressure gradient and velocity time integral ratio at baseline and 6-month follow-up).

5. Death (all-cause, cardiovascular or non-cardiovascular mortality).

6. Myocardial infarction.

7. Stroke (disabling or non-disabling) or transient ischaemic attack

8. Bleeding event (life-threatening or disabling, major bleeding or minor).

*All clinical endpoints are adjudicated according to the VARC- $2^{19}$ and the NeuroARC ${ }^{22}$ definitions

NeuroARC, Neurologic Academic Research Consortium; VARC-2, Valve Academic Research Consortium-2. 
Academic Research Consortium definitions. ${ }^{22}$ Detailed definitions of clinical endpoints are summarised in online supplemental appendix table 1 . The investigators in each centre should complete case report forms for all events and provide sufficient information for central review. All components of the primary and secondary endpoints are blindly adjudicated by an independent Clinical Event Committee (CEC).

After completion of the TAVR procedure, all study patients are monitored per institutional standard of care. The study subjects are followed at 1 month ( \pm 2 weeks), 3 months ( \pm 2 weeks) and 6 months $( \pm 1$ month). Data collected during all follow-up visits also include clinical symptoms, such as dyspnoea (New York Heart Association class), angina status (Canadian Cardiovascular Society class) and any related clinical events including rehospitalisation or unintended hospital visits. For compliance check, the investigator will keep track of investigational drug dispensed and/or administered to the subjects and it is for compliance calculation.

To confirm the occurrence of leaflet thrombosis of bioprosthetic valves, all subjects undergo 4-D, volumerendered cardiac CT at 6 months $( \pm 1$ month) after the TAVR. To evaluate the clinical effect of antithrombotic strategy and cerebral embolisation by leaflet thrombosis, we perform brain MRI at 1-7 days after TAVR and 6 months after initiating study drug administration. Transthoracic echocardiography is routinely performed at baseline, 1-7 days after immediate post-TAVR, 1 month and 6 months after initiating study drug administration. Standardised definitions of structural deterioration and valve failure are used for the echocardiographic imaging assessment of bioprosthetic valve dysfunction. ${ }^{23}$

\section{Acquisition and archive of cardiac CT and brain MRI}

A central imaging core lab (Asan Image Metrics; www.aimaicro.com) is in charge of image acquisition and archive. The image core lab establishes the standardised acquisition protocols of cardiac CT and brain MRI imaging through gathering all CT/MRI machines and acquisition protocols of cardiac CT and brain MRI in each participating site. All sites should be qualified for their imaging machines and capability to perform the standardised acquisition protocol by the imaging core lab. All CT/MRI images acquired from each site are anonymised and electronically transferred to a central server (AiCRO system; Asan Image Metrics, Seoul, Korea) for image archiving images and blinded independent image review. ${ }^{24}$

All cardiac CT scans are performed with a dedicated 4-D, volume-rendered CT acquisition protocol with intravenous contrast administration as mandated at each participating site. The archived CT images are reconstructed to generate the sagittal and coronal images (twochamber and three-chamber views) of the aortic root and volume-rendered en face view images of the device. Detailed information on acquisition and reconstruction methodology of cardiac CT is summarised in online supplemental appendix table 2 . The standardised cardiac
CT protocols comply with international expert consensus reports. ${ }^{25} 26$

All brain MRI scans are obtained including diffusionweighted imaging (DWI), fluid-attenuated inversion recovery (FLAIR), and T2-star gradient (GRE) sequences which are the important sequences for image endpoint. Other sequences such as localiser, T1-weighted image, T2-weighted image or MR angiography, can be allowed to use institutional protocols. The MRI sequences are in compliance with the 2018 American Heart Association/American Stroke Association guidelines. ${ }^{27}$ Detailed information on acquisition protocols of brain MRI is summarised in online supplemental appendix table 3 .

\section{Core laboratory image analyses}

An independent image review committee is organised by the central imaging core lab (Asan Image Metrics) for the analysis of CT and MRI data from the ADAPT-TAVR trial in a blinded fashion. Two cardiac radiologists analyse cardiac CT images, and two neuroradiologists evaluate brain MRI images in an independent and blinded manner. In cases of discrepancy, the adjudication was made by open discussion and consensus between radiologists and investigators. The adjudication variables are presence of valvular thrombosis and occurrence of new DWI-positive lesions, FLAIR-positive lesions, or GRE-positive lesions. The adjudication rates between readers and the rationale of adjudication should be recorded. The detailed items on the image analysis of cardiac CT and brain MR images are summarised in online supplemental appendix table 4,5 , respectively.

The cardiac CT images are analysed for presence of valve thrombosis, presence of leaflet thickening, leaflet motion based on opening limitation, stent eccentricity $(\%)$ and calcification volume. ${ }^{28}$ Presence of valve thrombosis is checked when there are hypoattenuated abnormal lesion(s) attached at the one or more transcatheter heart valve leaflet, subvalvular area, supravalvular area or left ventricular outflow tract (LVOT). The location of valve thrombosis should be determined from one or more of the followings: leaflet, subvalcular area, supravalvular area and LVOT. Leaflet motion is assessed based on grade of opening limitation on a volume-rendered en face image of the aortic-valve prosthesis at maximal leaflet opening. Leaflet motion is categorised as normal, mildly reduced ( $<50 \%$ reduction), moderately reduced $(50 \%$ to $70 \%$ reduction), severely reduced ( $>70 \%$ reduction) or immobile (lack of motion) in at least one valve leaflet. We classified patients with mild or no restriction of leaflet motion as having normal leaflet motion. The stent eccentricity is defined as 1 (minimum stent diameter/maximum stent diameter) at the level of inflow, valvular area and outflow tract. If there is calcification, readers should measure the volume of calcification at the annulus or sinus or Valsalva level. Calcification can be measured using the threshold of CT numbers greater than 850 Hounsfield unit.

The brain MRI images are analysed for occurrence, number, and volume of new lesions on the 6-month DWI/ 
FLAIR and GRE images compared with baseline MRI (immediate post-TAVR), respectively. The new lesions on DWI or FLAIR may reflect ischaemic lesions due to thromboembolic events but also might be attributed to other non-specific lesions. The new lesions on GRE are regarded as new haemorrhagic lesions. The occurrence of new lesion is defined when a lesion is seen only on 6-month MRI and not on baseline MRI. The number of new lesions is counted based on new separate lesions on 6-month MRI. The volume is calculated as the sum of volumes of all separate new lesions on 6-month brain MRI.

\section{Neurological and neurocognitive function assessment}

All study subjects will undergo detailed neurological and neurocognitive function assessment at post-TAVR $(1-7$ days after TAVR and before discharge) and 6 months of study drug administration. Neurologic assessments include standard clinical scales (the National Institutes of Health Stroke Scale (NIHSS) and the modified Rankin Scale $(\mathrm{mRS}))$ and cognitive assessments include the Montreal Cognitive Assessment. Dedicated attending staff will be identified at each centre to perform the neurological and cognitive assessments; these subjects are NIHSS certified, trained in administration of the mRS and cognitive tests, and are blinded to brain MRI findings and treatment groups.

\section{Sample size estimation and statistical analyses}

Sample size was estimated to simultaneously meet the primary endpoint of the incidence of leaflet thrombosis on cardiac CT and meet the key secondary endpoint of the total new lesion number on brain MRI. Based on the results from RESOLVE and SAVORY registry, ${ }^{11}$ we assumed an incidence of subclinical leaflet thrombosis of $15 \%$ in the DAPT group and of $3 \%$ in the NOAC (edoxaban) group. Enrolment of 192 patients (96 patients in each arm) would provide the study with a statistical power of $80 \%$ to detect this difference with a two-sided significance level of 0.05 . Assuming $10 \%$ attrition rate of CT follow-up loss at 6 months, a total of 220 patients (110 patients per each arm) are finally planned. In similar setting of post-TAVR status, there are no benchmark MRI data at immediate post-TAVR and follow-up on which to base control arm assumption. Among the two landmark trials (The Claret Embolic Protection and Transcatheter Aortic Valve Implantation (CLEAN-TAVI) ${ }^{29}$ and Cerebral Protection in TAVR (SENTINEL) ${ }^{30}$ involving brain MRI at post-TAVR, the median number of new lesions in the entire brain (with reference of the control arm) at immediate post-TAVR was 16 (IQR 10-24) in the CLEAN-TAVI trial and 5 (IQR 2-10) in the SENTINEL trial. It is expected that the absolute new lesion number between 6 months and immediate post-TAVR would be lower than the lesions number between immediate post-TAVR and baseline (pre-TAVR). Thus, we assumed that the mean number of new lesions in the entire brain between 6 months and immediate post-TAVR would be approximately 10 . Our hypothesis for key secondary endpoint of brain DW-MRI is that the use of edoxaban would provide a $30 \%$ reduction in the number of positive DW MRI-perfused brain lesions following TAVR at 6 months relative to post-TAVR in the entire brain compared with the use of DAPT. This relative risk reduction was based on the clinical observation of prior registry ${ }^{11}$ and the assumption of trial with similar concept. ${ }^{31}$ Given an SD of 7, which was based on the value of the CLEAN-TAVI trial, for the measure and assuming a drop-out rate of $20 \%$, a total of 218 patients (109 patients per each group) was estimated for the study to have a power of $80 \%$ at a two-sided $\alpha$-level of 0.05 . To meet the predefined estimation of this key secondary endpoint, the final sample size was estimated as a total of 220 patients (110 patients per each arm).

The primary and secondary endpoint analyses are conducted on the full analysis set of all randomised patients according to the intention-to-treat principle. The Fisher's exact test is used to compare categorical variables. Continuous variables, presented as mean $\pm \mathrm{SD}$ or medians with IQRs as appropriate, are compared with the use of the Student's t-test or the Mann-Whitney U test. The key secondary endpoint, consisting of new median lesion number differences between the two randomised arms, was compared using the Wilcoxon rank sum test. A z-score for each neurocognitive function domain is calculated on the basis of normative mean $\pm \mathrm{SD}$ for each neurocognitive test. Change scores are calculated by subtracting immediate-post-TAVR scores from the 6-month postTAVR scores. Cumulative event curves are generated by means of the Kaplan-Meier method. The 95\% CI of the HR will be presented using a Cox model for survival analysis. Trial data are held by the trial coordination centre at the Asan Medical Center. Analyses will be performed by independent statistical analysts who was unaware of randomised drug. All P-p alues are two sided, and values $<0.05$ are considered statistically significant.

\section{Study committees}

The executive committee (EC) is composed of principal investigators of clinical sites and persons who will organise this study. The EC will be responsible for reviewing the final results, determining the methods of presentation and publication, and selection of secondary projects and publications. National lead investigators and academic experts are part of the steering committee and responsible for the protocol implementation and study recruitment. An independent data safety monitoring board (DSMB) has the responsibility of monitoring safety during the trial: the members of the DSMB will not be among those who directly control the sponsor of this study and periodically review the safety data according to a dedicated charter and make recommendations based on safety analyses, protocol deviation, imaging failures and 6-month follow-up reports. The CEC consists of interventional and non-interventional cardiologists who are also independent and blinded. The CEC is charged of the development of specific criteria used for the 
categorisation of clinical events in the study, which are based on the protocol and will adjudicate all suspected study endpoints as detailed in the specific charter.

\section{Ethics and dissemination}

This trial was performed in accordance with the Declaration of Helsinki, International Conference on Harmonisation, Good Clinical Practice guidelines, and applicable regulatory requirements. Ethic approval and informed consent form have been obtained from the Ethics Committee /Institutional Review Board of Asan Medical Center (approval number: 2017-1317) and the trial was also approved by National Institute of Food and Drug Safety Evaluation of Republic of Korea (approval number: 31511). The study background and main objective as well as potential benefits and risks will be fully explained to the participants and their families. All participants voluntarily signed a declaration of informed consent. We planned to disseminate the overall results of the study to the participants and the public, such as presenting primary results in the international scientific meeting and publicising our research in medical news and various academic lectures.

\section{DISCUSSION}

The ADAPT-TAVR trial is a randomised controlled trial to define optimal antithrombotic strategy using direct acting factor Xa inhibitor, edoxaban after TAVR with regards to prevention of leaflet thrombosis and cerebral embolisation. This trial will provide randomised evidences of the efficacy and safety of edoxaban-based anticoagulation strategy compared with DAPT strategy after successful TAVR without indication of chronic OAC.

Initially, safety concern has been raised after report of cardiac CT findings in patients who had stroke after TAVR from an ongoing clinical trial. ${ }^{10}$ Large-sized observational registry showed that subclinical leaflet thrombosis more frequently developed in TAVR (13\%) than in SAVR $(4 \%),{ }^{11}$ but recent reports from CT substudies of low-risk RCTs showed comparable incidences of leaflet thrombosis after TAVR and SAVR. ${ }^{13} 32$ In prior observation, OAC (both VKA and NOACs) was more effective than DAPT in prevention or treatment of subclinical leaflet thrombosis ( $4 \%$ vs $15 \%$ ), and clinically subclinical leaflet thrombosis was associated with increased rates of TIAs and strokes. ${ }^{11}$ Although there was limited evidence supporting the association of leaflet thrombosis and cerebral embolic events, the Food and Drug Administration (FDA) has raised the safety concerns of TAVR and has been closely monitoring this signal. ${ }^{33}$ The FDA also recommended that whether reduced leaflet motion was clinically meaningful for patients with TAVR, the loss of mobility of one or more leaflets detected by CT rendered the valve structurally dysfunctional and demands additional investigation. After such safety concern has been raised in several studies, ${ }^{10} 113435$ updated guidelines suggest that OAC within at least 3 months is reasonable considering the possibility of leaflet thrombosis. ${ }^{16}$ However, there still has been inadequate evidence to support these OAC recommendations in patients undergoing TAVR.

Until recently, the underlying mechanism of bioprosthetic valve thrombosis were not clearly determined. The implanted TAVR valve adds a prothrombotic environment, which might be related to perturbations in blood flow (ie, stagnant blood) and activation of various haemostatic factors within the neosinus, ${ }^{14}$ and this condition may favour subclinical thrombosis and valve haemodynamic deterioration. Moreover, some studies suggested that the intra-annular valves was more prone to higher risk of leaflet thrombosis than the supra-annular valve, ${ }^{11} 36$ which would be the rationale of stratified randomisation by type of TAVR valve (balloon-expandable or self-expandable) in this trial. Although it is still unknown whether post-TAVR produced-thrombi have a predominant platelet-related or thrombin-related origin, thrombin plays a key role in the formation of thromboembolic events; the mechanisms of platelet activation and coagulation are highly interdependent, with thrombin playing a central role in both pathways. ${ }^{37}$ Given that direct factor Xa inhibitors target specifically factor Xa and decrease the conversion of prothrombin to active thrombin, thereby diminishing fibrin formation and reducing coagulation and platelet activation, it might be reasonable to consider a systemic anticoagulation strategy with NOAC regimen to prevent subclinical leaflet thrombosis and reduce the long-term thromboembolic risk after TAVR.

In this context, a systematic anticoagulation strategy after TAVR should be tested in RCTs. Recently, the primary results from the Global Study Comparing a Rivaroxabanbased Antithrombotic Strategy to an Antiplatelet-based Strategy after TAVR to Optimise Clinical Outcomes (GALILEO) showed that NOAC strategy with rivaroxaban at a dose of $10 \mathrm{mg}$ (with low-dose aspirin for the first 3 months) was associated with higher risks of thromboembolic complications, bleeding events and mortality than DAPT strategy (low-dose aspirin with clopidogrel at a dose of $75 \mathrm{mg}$ for the first 3 months) in patients without an OAC indication after successful TAVR. ${ }^{38}$ In an imaging substudy of GALILEO, a rivaroxaban-based antithrombotic strategy was more effective than DAPT strategy in preventing subclinical leaflet motion abnormalities $(2.1 \%$ vs $10.9 \%) .{ }^{39}$ Unfortunately, these findings cannot recommend routine imaging for the detection of reduced leaflet motion or routine use of anticoagulation after TAVR for preventing leaflet motion abnormalities, given the unfavourable clinical outcomes with rivaroxaban. Subsequent reports from the POPular TAVI trial cohort $\mathrm{A}$ and $\mathrm{B}$ showed that aspirin or OAC alone was associated with a lower incidence of bleeding and similar risk of thromboembolic events as compared with dual therapy with clopidogrel. ${ }^{40}$ Regarding this important issue, an OAC strategy alone or NOAC strategy instead of VKA is actively being tested in another ongoing RCTs including the ADAPT-TAVR trial (ATLANTIS trial: NCT02664649, ENVISAGE-TAVI AF: NCT02943785, and AVATAR: NCT02735902). The release of the key 
results of such consecutive trial may provide compelling evidence to resolve the clinical unmet need for optimal antithrombotic strategy in the routine clinical practice of TAVR. In addition, the potential preventive role of anticoagulation with NOAC for preventing leaflet thrombosis and cerebral embolisation after TAVR, which was not yet confirmed by RCTs, will be supported by the primary results of the ADAPT-TAVR trial.

It should be acknowledged that this study has several limitations. First, bias in event ascertainment cannot be ruled out given the open-label trial design. Second, the ADAPT-TAVR trial has adopted the surrogate imaging outcome as the primary and key secondary endpoints. Therefore, our key findings based on imaging modalities may not fully support the compelling clinical rationale with regard to efficacy and safety of NOAC strategy. Third, our trial was underpowered to detect any clinically relevant differences in clinical outcomes between two treatment strategies. Finally, we excluded patients with an established indication for OAC, which might be at least one-third of the TAVR population. Thus, our findings cannot be directly extrapolated to such population.

\section{Trial status}

The ADAPT-TAVR trial is planned to complete the 3-year enrolment period for the prespecified 220 subjects from the five participating centres. The first patient was enrolled on March 2018, and 200 patients have been enrolled until October 2020. Enrolment may be completed approximately by the end of 2020. Primary results of the ADAPTTAVR trial will be available by late-term of 2021 .

\section{Author affiliations}

${ }^{1}$ Division of Cardiology, Asan Medical Center, Songpa-gu, Seoul, The Republic of Korea

${ }^{2}$ Asan Image Metrics, Clinical Trial Center, Asan Institute for Life Sciences, Asan Medical Center, Songpa-gu, Seoul, The Republic of Korea

${ }^{3}$ Department of Radiology and Research Institute of Radiology, Asan Medical Center, Songpa-gu, Seoul, The Republic of Korea

${ }^{4}$ Division of Cardiology, Department of Medicine, Queen Mary Hospital, Pok Fu Lam, Hong Kong

${ }^{5}$ Heart Center, Cheng Hsin General Hospital, Taipei, Taiwan

${ }^{6}$ Division of Cardiology, Department of Internal Medicine, National Taiwan University Hospital, Taipei, Taiwan

${ }^{7}$ Division of Cardiology, Department of Internal Medicine, Hsin-Chu Branch, National Taiwan University Hospital, Hsin-Chu, Taiwan

${ }^{8}$ Department of Cardiology, CHA Bundang Medical Center, Seongnam, Gyeonggi-do, The Republic of Korea

${ }^{9}$ Department of Neurology, Asan Medical Center, Songpa-gu, Seoul, The Republic of Korea

${ }^{10}$ Department of Biostatistics, Asan Medical Center, Songpa-gu, Seoul, The Republic of Korea

Acknowledgements We thank the staff of the ADAPT-TAVR trial, the other members of the cardiac catheterisation laboratories and the heart team at the participating centres, and the study coordinators for their efforts in collecting clinical data and ensuring the accuracy and completeness of the data.

Contributors Contributors Study conception and design: D-WP, HP, D-YK, J-MA and S-JP; drafting of the study protocol: HP, KWK and D-WP; critical revision of the study protocol for important intellectual content: HP, D-YK, J-MA, KWK, AYTW, SCCL, W-HY, JW, Y-TL, H-LK, M-SL, T-YK, W-JK, SHK, EK, D-HK, HJK, DHY, J-WK, SCJ, J-HL, S-CY, S-JP and D-WP; statistical expertise: S-CY; obtaining of research funding: D-WP; administrative, technical, or logistic support: HP, D-YK, J-MA, KWK, AYTW, SCCL, W-
HY, JW, Y-TL, H-LK, M-SL, T-YK, W-JK, SHK, EK, D-HK, HJK, DHY, J-WK, SCJ, J-HL, S-CY, S-JP and D-WP; acquisition of data: HP, D-YK, J-MA, KWK, AYTW, SCCL, W-HY, JW, Y-TL, H-LK, M-SL, T-YK, W-JK, SHK, EK, D-HK, HJK, DHY, J-WK, SCJ, J-HL, S-CY, S-JP and D-WP. All authors approved the final manuscript.

Funding The ADAPT-TAVR trial is partly funded by Daiichi Sankyo Inc. and CardioVascular Research Foundation (CVRF, Seoul, Korea) (grant number: AMCCV 2017-08). None of the study leadership accepted any compensation for their roles in this study, other than expenses. The principal investigators accept responsible for the design and conduct of this study, all study analyses, and the drafting and editing of all manuscripts.

Competing interests None declared.

Patient consent for publication Obtained.

Provenance and peer review Not commissioned; externally peer reviewed.

Supplemental material This content has been supplied by the author(s). It has not been vetted by BMJ Publishing Group Limited (BMJ) and may not have been peer-reviewed. Any opinions or recommendations discussed are solely those of the author(s) and are not endorsed by BMJ. BMJ disclaims all liability and responsibility arising from any reliance placed on the content. Where the content includes any translated material, BMJ does not warrant the accuracy and reliability of the translations (including but not limited to local regulations, clinical guidelines, terminology, drug names and drug dosages), and is not responsible for any error and/or omissions arising from translation and adaptation or otherwise.

Open access This is an open access article distributed in accordance with the Creative Commons Attribution Non Commercial (CC BY-NC 4.0) license, which permits others to distribute, remix, adapt, build upon this work non-commercially, and license their derivative works on different terms, provided the original work is properly cited, appropriate credit is given, any changes made indicated, and the use is non-commercial. See: http://creativecommons.org/licenses/by-nc/4.0/.

ORCID iD

Duk-Woo Park http://orcid.org/0000-0001-6643-0239

\section{REFERENCES}

1 Leon MB, Smith CR, Mack M, et al. Transcatheter Aortic-Valve Implantation for Aortic Stenosis in Patients Who Cannot Undergo Surgery. N Engl J Med Overseas Ed 2010;363:1597-607.

2 Smith CR, Leon MB, Mack MJ, et al. Transcatheter versus surgical aortic-valve replacement in high-risk patients. $N$ Engl J Med 2011;364:2187-98.

3 Makkar RR, Fontana GP, Jilaihawi H, et al. Transcatheter aortic-valve replacement for inoperable severe aortic stenosis. N Engl J Med 2012;366:1696-704.

4 Kodali SK, Williams MR, Smith CR, et al. Two-Year outcomes after transcatheter or surgical aortic-valve replacement. N Engl J Med 2012;366:1686-95.

5 Adams DH, Popma JJ, Reardon MJ, et al. Transcatheter aorticvalve replacement with a self-expanding prosthesis. $N$ Engl J Med Overseas Ed 2014;370:1790-8.

6 Leon MB, Smith CR, Mack MJ, et al. Transcatheter or surgical aortic-valve replacement in intermediate-risk patients. $N$ Engl J Med 2016;374:1609-20.

7 Reardon MJ, Van Mieghem NM, Popma JJ, et al. Surgical or transcatheter aortic-valve replacement in intermediate-risk patients. N Engl J Med 2017;376:1321-31.

8 Mack MJ, Leon MB, Thourani VH, et al. Transcatheter aortic-valve replacement with a Balloon-Expandable valve in low-risk patients. $N$ Engl J Med 2019;380:1695-705.

9 Popma JJ, Deeb GM, Yakubov SJ, et al. Transcatheter aortic-valve replacement with a self-expanding valve in low-risk patients. $N$ Engl J Med 2019;380:1706-15.

10 Makkar RR, Fontana G, Jilaihawi $\mathrm{H}$, et al. Possible subclinical leaflet thrombosis in bioprosthetic aortic valves. $N$ Engl J Med 2015;373:2015-24.

11 Chakravarty T, Søndergaard L, Friedman J, et al. Subclinical leaflet thrombosis in surgical and transcatheter bioprosthetic aortic valves: an observational study. Lancet 2017;389:2383-92.

12 Rashid HN, Gooley RP, Nerlekar N, et al. Bioprosthetic aortic valve leaflet thrombosis detected by multidetector computed tomography is associated with adverse cerebrovascular events: a meta-analysis of observational studies. Eurolntervention 2018;13:e1748-55.

13 Makkar RR, Blanke P, Leipsic J, et al. Subclinical Leaflet Thrombosis in Transcatheter and Surgical Bioprosthetic Valves: PARTNER 
3 Cardiac Computed Tomography Substudy. J Am Coll Cardiol 2020;75:3003-15.

14 Puri R, Auffret V, Rodés-Cabau J. Bioprosthetic valve thrombosis. J Am Coll Cardiol 2017;69:2193-211.

15 Capodanno D, Angiolillo DJ. Antithrombotic therapy for prevention of cerebral thromboembolic events after transcatheter aortic valve replacement: evolving paradigms and ongoing directions. JACC Cardiovasc Interv 2017;10:1366-9.

16 Nishimura RA, Otto CM, Bonow RO. AHA/ACC focused update of the 2014 AHA/ACC guideline for the management of patients with valvular heart disease: a report of the American College of Cardiology/American heart association Task force on clinical practice guidelines. Circulation 2017;2017:e1159-95.

17 Giugliano RP, Ruff CT, Braunwald E, et al. Edoxaban versus warfarin in patients with atrial fibrillation. N Engl J Med 2013;369:2093-104.

18 Büller HR, Décousus H, Middeldorp S. Edoxaban versus warfarin for the treatment of symptomatic venous thromboembolism. N Engl J Med 2013;369:1406-15.

19 Kappetein AP, Head SJ, Généreux P, et al. Updated standardized endpoint definitions for transcatheter aortic valve implantation: the valve academic research Consortium-2 consensus document. J Am Coll Cardiol 2012;60:1438-54

20 Yoon Y-H, Ahn J-M, Kang D-Y, et al. Incidence, predictors, management, and clinical significance of new-onset atrial fibrillation after transcatheter aortic valve implantation. Am J Cardiol 2019;123:1127-33.

21 Kappetein AP, Head SJ, Généreux P, et al. Updated standardized endpoint definitions for transcatheter aortic valve implantation: the valve academic research Consortium-2 consensus document (VARC2). Eur J Cardiothorac Surg 2012;42:S45-60.

22 Lansky AJ, Messé SR, Brickman AM, et al. Proposed standardized neurological endpoints for cardiovascular clinical trials. J Am Coll Cardiol 2017;69:679-91.

23 Capodanno D, Petronio AS, Prendergast B, et al. Standardized definitions of structural deterioration and valve failure in assessing long-term durability of transcatheter and surgical aortic bioprosthetic valves: a consensus statement from the European association of percutaneous cardiovascular interventions (EAPCl) endorsed by the European Society of cardiology (ESC) and the European association for Cardio-Thoracic surgery (EACTS). Eur Heart J 2017;38:3382-90.

24 Shin Y, Kim KW, Lee AJ, et al. A good practice-compliant clinical trial imaging management system for multicenter clinical trials: development and validation study. JMIR Med Inform 2019;7:e14310.

25 Otto CM, Kumbhani DJ, Alexander KP. Acc expert consensus decision pathway for transcatheter aortic valve replacement in the management of adults with aortic stenosis: a report of the American College of cardiology Task force on clinical expert consensus documents. J Am Coll Cardiol 2017;2017:1313-46.

26 Achenbach S, Delgado V, Hausleiter J, et al. SCCT expert consensus document on computed tomography imaging before transcatheter aortic valve implantation (TAVI)/transcatheter aortic valve replacement (TAVR). J Cardiovasc Comput Tomogr 2012;6:366-80

27 Powers WJ, Rabinstein AA, Ackerson T. Guidelines for the early management of patients with acute ischemic stroke: a guideline for healthcare professionals from the American heart Association/ American stroke association. Stroke 2018;2018:e46-110.

$28 \mathrm{Koo} \mathrm{HJ}$, Choe J, Kang D-Y, et al. Computed tomography features of Cuspal thrombosis and subvalvular tissue Ingrowth after transcatheter aortic valve implantation. Am J Cardiol 2020;125:597-606.

29 Haussig S, Mangner N, Dwyer MG, et al. Effect of a cerebral protection device on brain lesions following transcatheter aortic valve implantation in patients with severe aortic stenosis: the CLEAN-TAVI randomized clinical trial. JAMA 2016;316:592-601.

30 Kapadia SR, Kodali S, Makkar R, et al. Protection against cerebral embolism during transcatheter aortic valve replacement. J Am Coll Cardiol 2017;69:367-77.

31 Collet J-P, Berti S, Cequier A, et al. Oral anti-Xa anticoagulation after trans-aortic valve implantation for aortic stenosis: the randomized Atlantis trial. Am Heart J 2018;200:44-50.

32 Blanke P, Leipsic JA, Popma JJ, et al. Bioprosthetic Aortic Valve Leaflet Thickening in the Evolut Low Risk Sub-Study. J Am Coll Cardiol 2020;75:2430-42.

33 Laschinger JC, Wu C, Ibrahim NG, et al. Reduced Leaflet Motion in Bioprosthetic Aortic Valves--The FDA Perspective. N Engl J Med 2015;373:1996-8.

34 Hansson NC, Grove EL, Andersen HR, et al. Transcatheter aortic valve thrombosis: incidence, predisposing factors, and clinical implications. J Am Coll Cardiol 2016;68:2059-69.

35 Pache G, Schoechlin S, Blanke P, et al. Early hypo-attenuated leaflet thickening in balloon-expandable transcatheter aortic heart valves. Eur Heart J 2016;37:2263-71.

36 Rashid HN, Nasis A, Gooley RP, et al. The prevalence of computed tomography-defined leaflet thrombosis in intra- versus supra-annular transcatheter aortic valve prostheses. Catheter Cardiovasc Interv 2018:92:1414-6.

37 Depta JP, Bhatt DL. New approaches to inhibiting platelets and coagulation. Annu Rev Pharmacol Toxicol 2015;55:373-97.

38 Dangas GD, Tijssen JGP, Wöhrle J, et al. A controlled trial of rivaroxaban after transcatheter aortic-valve replacement. $N$ Engl $J$ Med 2020;382:120-9.

39 De Backer O, Dangas GD, Jilaihawi $\mathrm{H}$, et al. Reduced leaflet motion after transcatheter aortic-valve replacement. N Engl J Med 2020;382:130-9.

40 Brouwer J, Nijenhuis VJ, Delewi R, et al. Aspirin with or without clopidogrel after transcatheter aortic-valve implantation. N Engl J Med 2020;383:1447-57.

41 Nijenhuis VJ, Brouwer J, Delewi R, et al. Anticoagulation with or without clopidogrel after transcatheter aortic-valve implantation. $N$ Engl J Med 2020;382:1696-707. 